\title{
El paisaje sonoro en la Odisea
}

\section{Luis Calero}

\section{(Autonomous University of Madrid)}

\section{Soundscape in Odyssey}

\begin{abstract}
This article examines the way of expressing the world of sounds in Odyssey, which developes through the lexicon it employs. In this way, we analyze the contexts in which some natural elements are named, as well as their sounds and characteristics, and those of certain physical spaces as they are treated in the poem, with the intention of generating, as much as possible, the universe of sounds that allows to contextualize the poem from an aural point of view. The methodology is based on the linguistic concept of acoustic image that Saussure developed in the late $19^{\text {th }}$ century and follows Schafer's most recent tradition in his 1993 work on soundscape. Winds, sea, storms, palaces and voice will be analyzed in this paper.
\end{abstract}

\section{Keywords}

acoustic image; soundscape; Odyssey; Homer 


\section{Preliminares}

Los estudios sobre sonido y escucha como funciones de la historia y la cultura tienden a tratar de comprender los preceptos y prácticas sonoros como parte de la ecología de un auditorio que se basa en las relaciones culturales, industriales, científicas y tecnológicas entre música, habla, ruido y silencio. De este modo, los estudios sobre sonido adoptan una perspectiva más amplia que las disciplinas musicales en sí mismas. Se examina la música como un mero aspecto, importante, eso sí, de un espectro sonoro y auditivo mucho más amplio (Devine 2014).

No obstante, en lingüística esto no es novedoso. Saussure denominó en 1878 imagen acústica a aquella imagen mental que cada uno de nosotros oímos en nuestra cabeza ante determinada información no necesariamente audible y de ahí que también se llame imagen sonora. Este sonido se diferencia del material y físico, para convertirse en su huella psíquica.

La idea de Paisaje Sonoro, traducción del inglés soundscape (de sound + landscape), surge a partir del equipo de trabajo liderado por el compositor Raymond Murray Schafer en la Universidad Simon Fraser (Burnaby, Canadá). Se define como una disciplina que intenta distinguir y estudiar el universo sonoro de nuestro entorno, para, llegado el caso, poder reconstruir un paisaje sonoro a través de eventos escuchados y no necesariamente vistos. Schafer fundó el World Soundscape Project a finales de los 60 y el World Forum for Acustic Ecology en 1993. Este compositor, junto con otros investigadores, como Barry Truax y Hildegard Westercamp, ha publicado sus trabajos (Schafer 1969, 1970, 1977) sobre paisaje sonoro y ecología acústica (Soundscape Studies). Su metodología de investigación se ha extendido de manera progresiva a otras disciplinas, como la psicoacústica, el urbanismo, la música y las ciencias sociales. Uno de sus objetivos es fijar a través de documentos sonoros distintos espacios naturales que están en peligro de extinción sonora por efecto de la contaminación, de la deforestación del planeta, etc., a modo de un patrimonio sonoro inmaterial de la humanidad. No siempre es fácil imaginar los sonidos extinguidos del pasado. No obstante, algunos trabajos son dignos de ser mencionados a este respecto, como Nooter $(2012,2017)$, autora que se ha adentrado en la sonoridad de la tragedia de Esquilo y de Sófocles como espectáculo en directo y que más adelante coordinó un volumen (Butler \& Nooter 2019) en que diversos investigadores colaboran con trabajos dedicados al sentido del oído en la Antigüedad y al sonido del Mundo Antiguo. Uno de ellos (Montiglio 2019) será citado más adelante, por estar directamente relacionado con este artículo.

Entre la bibliografía específica no encontramos una definición única para paisaje sonoro/soundscape, pero se suele hacer hincapié en la experiencia perceptiva de un entorno dado por aquellos que forman parte de él. Este mundo sonoro refleja las condiciones sociales, tecnológicas y naturales de un espacio, definiendo para sus habitantes un sentido del lugar, que condiciona su comportamiento y los llega a identificar de manera acústica. Por esta razón, el paisaje sonoro suele ser analizado dentro de los parámetros tanto de manifestación de un lugar como de un medio a través del cual se reafirma el significado social de dicho espacio (Hill 2014). 
En consecuencia, ¿es lícito plantearse si esta metodología de estudio se puede aplicar a la literatura? ¿Dan los textos la información necesaria para poder imaginar el entorno sonoro de sus personajes? ¿Podemos hacer este estudio en el repertorio épico? Creemos que la respuesta es sí, aunque con matices, como se podrá ver en las conclusiones del artículo.

El estudio de la música en Homero se ha hecho desde distintas perspectivas. Algunos autores dedicaron a mediados del siglo XX unas páginas para dar leves pinceladas sobre la cuestión. Sachs (1940: pp. 129-130) habló brevemente de la forminge y la kítharis, los dos únicos instrumentos de cuerda habitualmente citados en Homero, en cuyos poemas también se menciona el auló únicamente en dos ocasiones: Il.10.113, cuando, acompañado de siringes, pone música a la velada de los troyanos; y en Il.18.495, en que forma parte de las celebraciones de una boda descrita en el escudo de Aquiles. West (1994: p. 82) matiza el detalle de que este instrumento no aparece en las situaciones en que uno podría esperarlo, como lamentos por la muerte de los héroes o indicaciones estratégicas en las batallas. Sin embargo, en 1943, este mismo autor obvió el tema, al igual que Henderson (1957), que tampoco dedica ni siquiera unas líneas a la música en la épica.

Ya en los años 70, Michaelides (1978: s.uu.) dedica sendas entradas a kítharis y a forminge, para explicar brevemente su historia, fuentes y organología con detalle. Por esas mismas fechas, Comotti (1986: pp. 4-5) trató muy por encima la cuestión musical épica. Tampoco West (1994) dedica especial atención a la información musical contenida en los poemas homéricos o en el repertorio épico. Si acaso lo nombra de pasada aquí y allá. Barker (1989: pp. 18-46) sí ha hecho un trabajo algo más concienzudo, recogiendo una parte importante de aquellos momentos en que Homero, así como también Hesíodo y los Himnos Homéricos (Valverde 1997), dibujan escenas musicales, pero aborda su estudio desde un punto de vista descriptivo y meramente acumulativo. La monografía de García López, Pérez Cartagena y Redondo Reyes dedica pocas páginas a Homero de manera específica (2012: pp. 70-73), así como algunas más a Hesíodo y a los Himnos Homéricos. Por último, Franklin (2015) estudia el mundo épico griego, pero lo hace desde una perspectiva que le permite acceder a la investigación de este repertorio en Chipre, de manera que es un importante complemento para completar una visión general de la cuestión.

$\mathrm{El}$ acercamiento a las técnicas compositivas y musicales parte principalmente de las contribuciones de Parry y Lord, aglutinadas en la monografía de este último (1960), y ha cobrado especial madurez a partir de los estudios de Danek \& Hagel (1995), que abordan el estudio desde las cuestiones relativas a los acentos de la lengua griega antigua. Hagel (2008) lo hace desde una interesante perspectiva arqueomusical.

Algunos artículos y capítulos de libros destacables sobre la música en Homero son West (1981), Montero Honorato (1988), García López (1997), Snoj (2007), Río Torres-Murciano (2014) o Montiglio (2019). Esta última aborda el estudio del sentido físico del oído en el contexto del episodio de las Sirenas, tanto en Homero como en la posterioridad romana y tardía. 


\section{Metodología}

En este artículo, se propone el análisis de los recursos léxicos empleados en la Odisea, investigando los contextos en que se tratan los elementos naturales, así como sus sonidos y las características que los describen. De esta manera, se contextualizará auditivamente la épica homérica, aunque, dado que el volumen de información es enorme, para esta ocasión se ha estudiado únicamente la Odisea, un poema si acaso más paisajístico, más de interiores y más descriptivo en general que la Ilíada. La edición empleada es la de Thomas W. Allen, tomos $3\left(1985^{14}[=1908]\right)$ y $4\left(1985^{13}[=1908]\right)$, Oxford, OCT. La traducción sigue, en la mayoría de los casos, la de Pedro C. Tapia Zúñiga para la Bibliotheca Scriptorum Graecorum et Romanorum Mexicana, de la Universidad Nacional Autónoma de México (edición bilingüe, 2014², reimpr. 2017), y la de José M. Pabón, de la Biblioteca Clásica Gredos (1982). No obstante, en ocasiones nos apartamos de sus versiones. En esos casos, la traducción será propia.

Homero es un autor rico en la expresión de los sonidos. Compone escenarios que no solo describen espacios geográficos o localizaciones concretas, sino que también reflejan las emociones de los personajes en cada momento. El vocabulario y el contexto en que este se aplica pueden determinar a su vez diversos aspectos de los elementos que se analizarán. Así, por ejemplo, el ruido del trueno se puede encontrar en múltiples ocasiones,

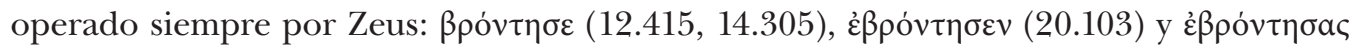

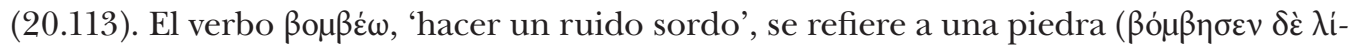

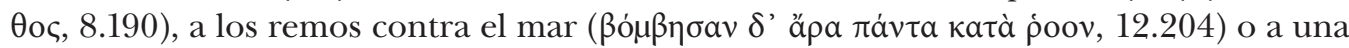

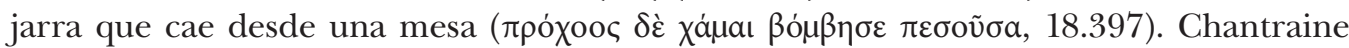
(1968: s.u.) y Beekes (2010: s.u.) suponen un origen onomatopéyico para esta familia léxica.

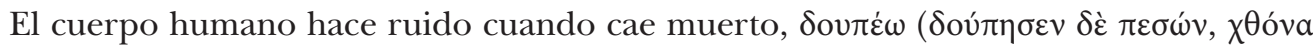

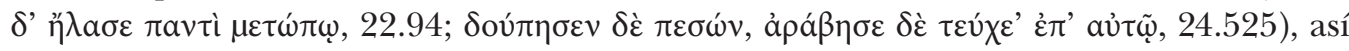
como el correspondiente $\delta$ oṽ $\pi$ ç, 'estruendo', se asocia con el que producen los huma-

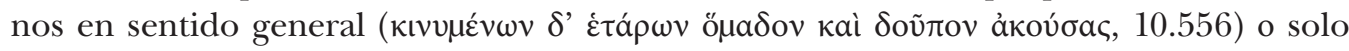

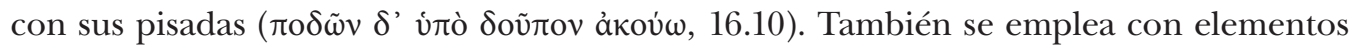

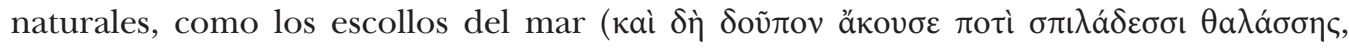

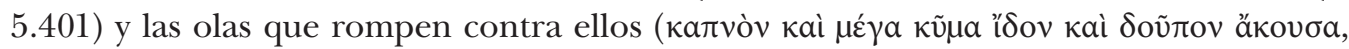
12.202). Este oleaje 'ruge' ( $\rho$ ó $\left.\chi \theta \varepsilon,{ }^{1} 5.402,12.60\right)$ o también retumba mientras 'vomita'

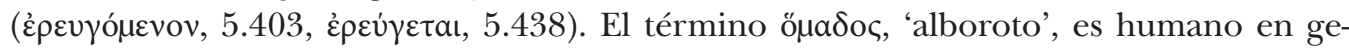

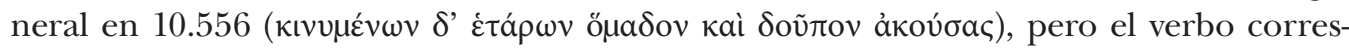
pondiente, ó $\mu a \delta \varepsilon \dot{\varepsilon} \omega$ 'armar alboroto', está relacionado en exclusiva con los pretendientes

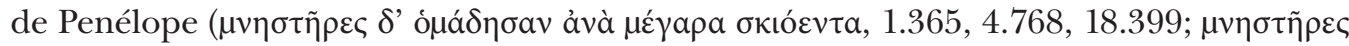

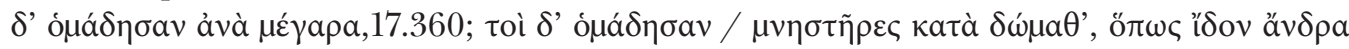
$\pi \varepsilon \sigma o ́ v \tau \alpha, 22.21){ }^{2}$

1 Término sin etimología conocida según Chantraine (1968: s.u.) y Beekes (2010: s.u.).

2 Chantraine (1968: s.u.) y Beekes (2010: s.u.) lo relacionan con el sánscrito samád-, 'combate', sobre la raíz de *som-, lo cual resulta bastante apropiado para este contexto de lucha entre los aspirantes a sustituir a Odiseo. 
En esta ocasión, detallaremos los datos obtenidos a partir del análisis léxico de los sonidos que producen los vientos, el mar, las tormentas, los palacios y, por último, la voz en la Odisea.

\section{El viento}

Uno de los términos que se emplea en la Odisea cuando se habla del viento es, lógica-

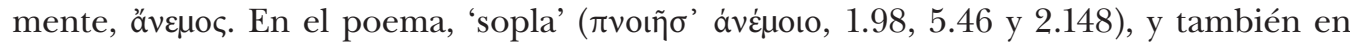

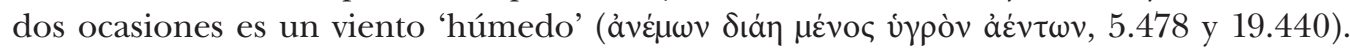
En 12.313, se matiza el sonido de este elemento cuando Zeus ordena que sople con

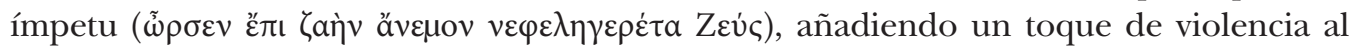
sonido que uno imagina.

El viento que sopla no siempre es bueno. Homero indica que puede ser 'malo' (åvenóv

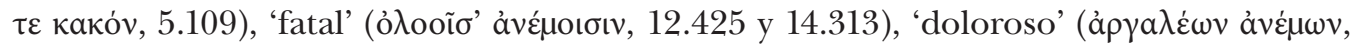

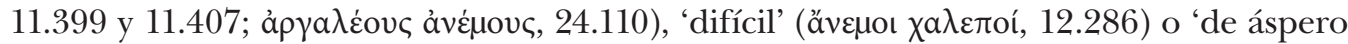

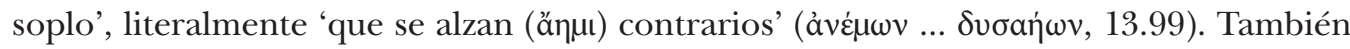
en nuestro texto el viento tiene voz propia, aguda y potente, como en 3.289, donde el viento es 'silbante, penetrante, agudo, potente' ( $\lambda_{\imath} \gamma \varepsilon \dot{\varepsilon} \omega \nu \delta$ ' ảvé $\left.\mu \omega v\right)$ o 'bramador' en 10.20 ( $\beta v \kappa \tau \alpha \dot{\alpha} \omega v$ àvé $\mu \omega v)$.

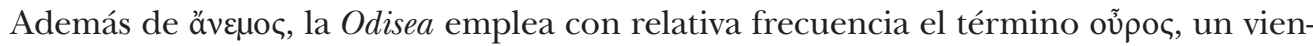
to que se muestra siempre favorable, cuya intervención genera buena fortuna. ${ }^{3}$ Es un viento constante, intenso, pero calmado. Es ese tipo de vientos 'que de naves son escolta

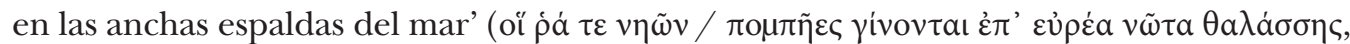

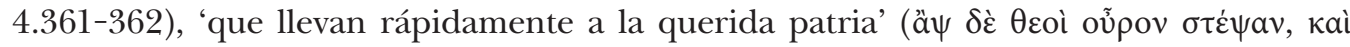

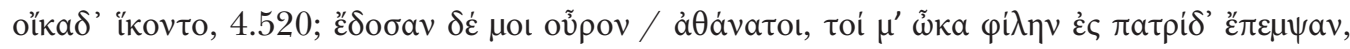
4.585-586, 17.148-149), porque asisten a las naves por la popa, para que puedan llegar

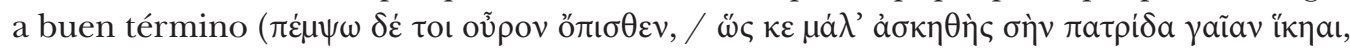

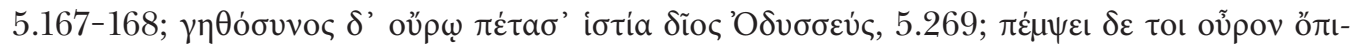

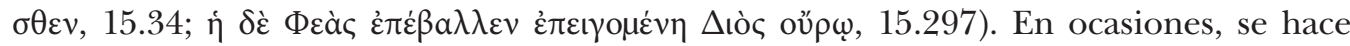

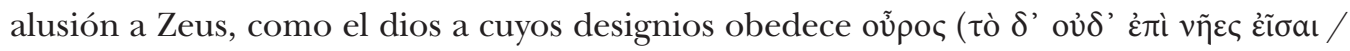

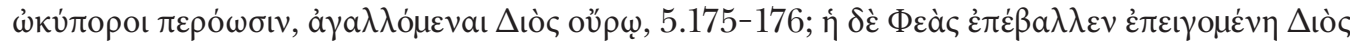

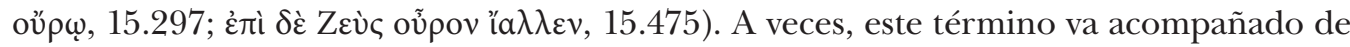
adjetivos que enriquecen sus cualidades sonoras. Así, puede ser 'suave' como una brisa

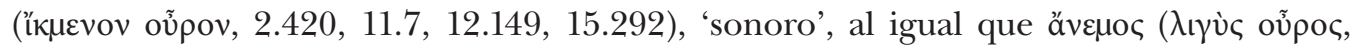

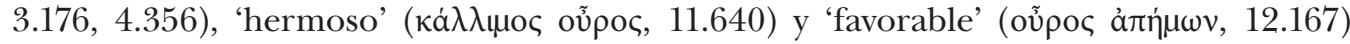

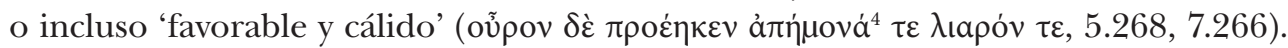

Además de estos vientos, a menudo se encuentran personificados en el Euro, el Noto, el Céfiro y el Bóreas, que pueden actuar 'todos de manera conjunta' ( $\pi \alpha v \tau o i \omega v$ ảvé $\mu \omega v$,

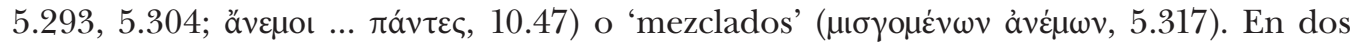

3 Chantraine (1968: s.u.) 'qui marche, qui avance avec vous'.

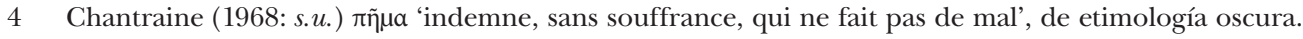


ocasiones, no obstante, aparecen los cuatro vientos nombrados individualmente ( $\sigma$ v̀v $\delta$ '

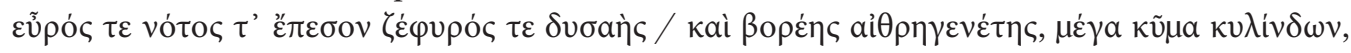

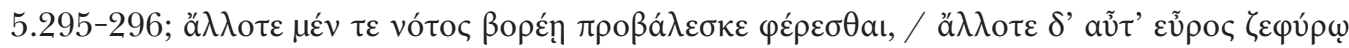

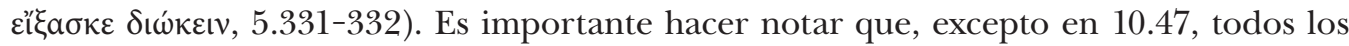
ejemplos pertenecen al libro $\mathrm{V}$ de la Odisea, aquel en el que Odiseo se prepara para abandonar la isla de Calipso antes de llegar a Esqueria, el país de los feacios, un episodio en que los vientos, sus acciones y su energía son parte importante del contexto sonoro del canto.

Si nos centramos en cada uno de ellos, podemos observar que el Euro, el viento del

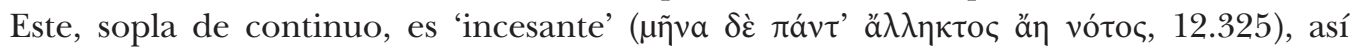

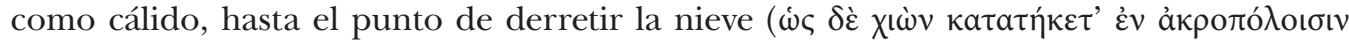

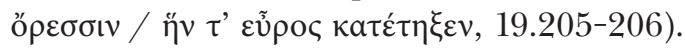

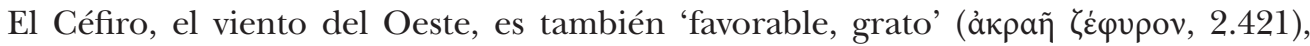

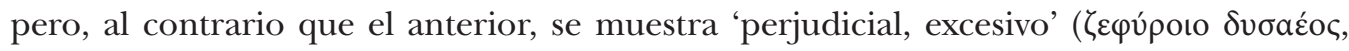

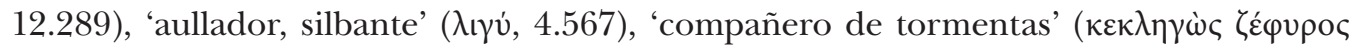

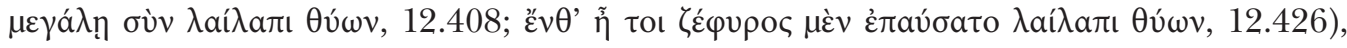

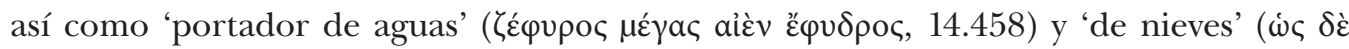

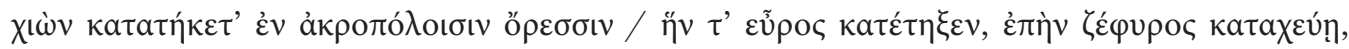
19.205-206).

El Bóreas, el viento del Norte, también es turbulento, frío y violento, pero, no obstante, se muestra a veces favorecedor. Se trata de un fenómeno turbulento, frío y violento,

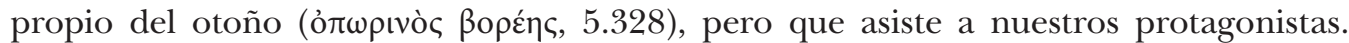

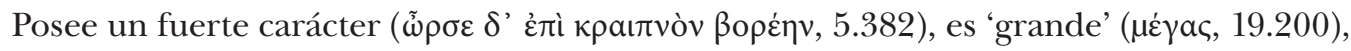

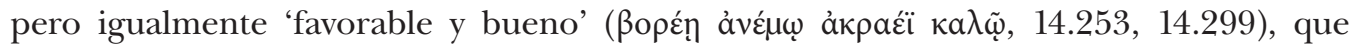

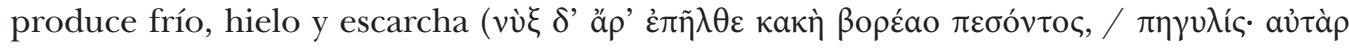

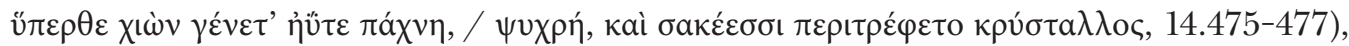

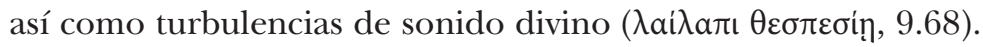

Por último, el Noto, el viento del Sur, es descrito como un viento 'incesante' ( $\mu$ ñva

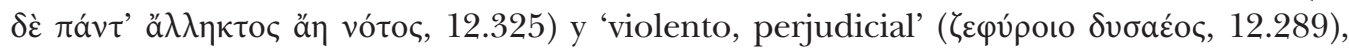

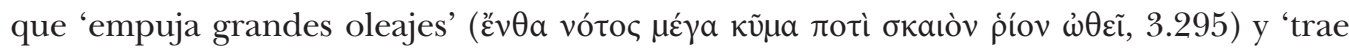

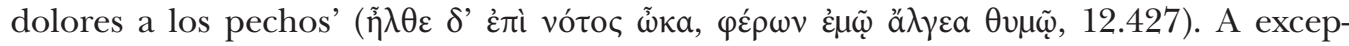
ción del ejemplo del Canto III, el resto pertenece al Canto XII, en el que se narran las desventuras de Odiseo y su tripulación con las Sirenas, Escila, Caribdis, etc. Este canto construye un cuadro sonoro bastante ilustrativo de los azares que atraviesan Odiseo y su tripulación.

Por último, cualquiera de estos vientos también puede ser huracanado. El término

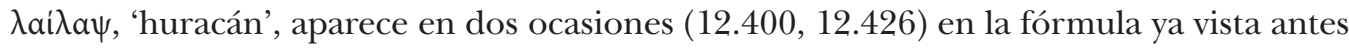

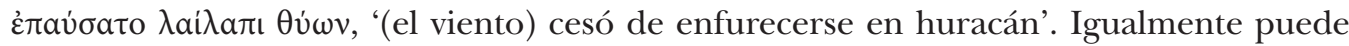

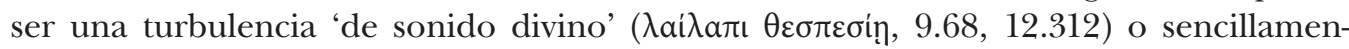

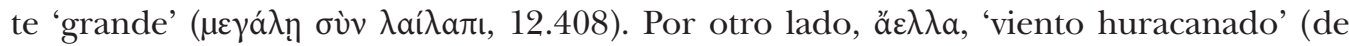
ă $\mu \mathrm{u})$, aparece en siete ocasiones en el poema (3.283, 3.320, 5.292, 5.304, 8.409, 14.383 y 19.189), pero nunca asociado a ningún sonido que se pueda esperar del fenómeno atmosférico que describe. 


\section{El mar}

El primer término al que uno acude cuando quiere investigar cómo expresar los ruidos del mar es, lógicamente, $\theta a ́ \lambda a \sigma \sigma \alpha$. Los setenta y ocho ejemplos de este término en el poema describen la anchura, su profundidad, la localización geográfica del mar como punto de partida o de llegada de los protagonistas y sus naves o sirven para ubicar playas y puntos geográficos; excepto en 13.85 y 13.220, en que se emplea la fórmula

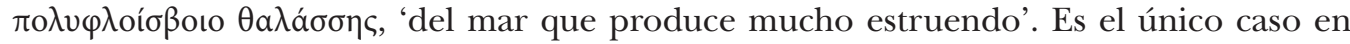
que este elemento se asocia a sonido.

El término å $\lambda \varsigma$, 'mar' aparece en el poema en cincuenta y ocho ocasiones, pero solo en

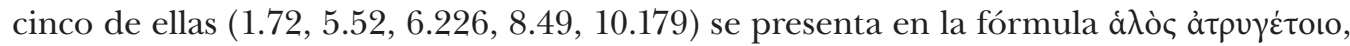
'del mar que se agita incansable', que ofrece contenido sonoro implícito. ${ }^{5}$

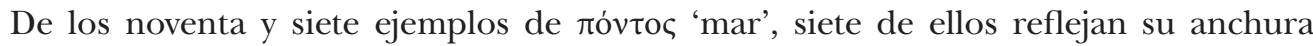
(1.297, 2.295, 4.498, 4.552, 12.293, 12.401, 24.118); diez, su cualidad brumosa (2.263, $3.105,3.294,4.482,5.164,5.281,8.568,12.285,13.150,13.176)$; siete, la ondulación de sus olas $(4.354,4.425,4.570,5.352,6.204,11.253,19.277)$; doce, su color vinoso al atardecer (1.183, 2.421, 3.286, 4.474, 5.132, 5.221, 5.349, 6.170, 7.250, 12.388, 19.172, 19.274); nueve lo describen lleno de peces (4.381, 4.390, 4.424, 4.470, 4.516, 5.420, 9.83, 10.458, 10.540); uno hace hincapié en sus grandes fauces (3.158); dos, en la infinitud de su extensión $(4.510,10.195)$ y otros dos, en su color violáceo $(5.56,11.107)$. Dejamos para el final los siete momentos $(2.370,5.84,5.140,5.158,7.79,13.419,17.289)$ en que se emplea

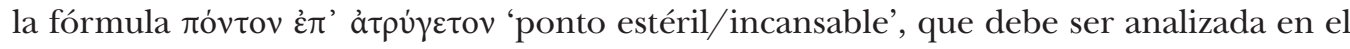

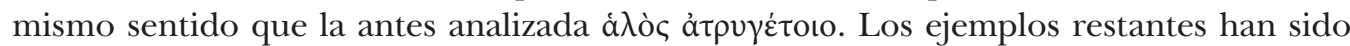
desechados por no dar ningún tipo de indicación en ningún sentido.

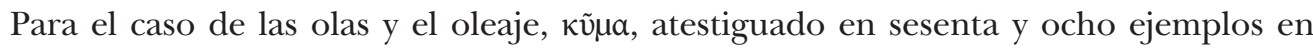
total, tan solo nueve de ellos enmarcan el término auditivamente. Se habla del tamaño de las olas, lo cual ya implica el ruido que producen, de manera que pueden ser 'gran-

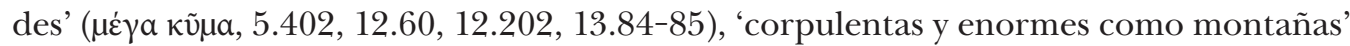

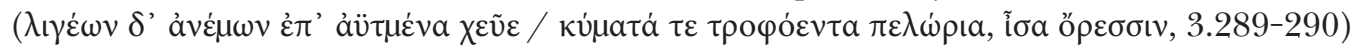

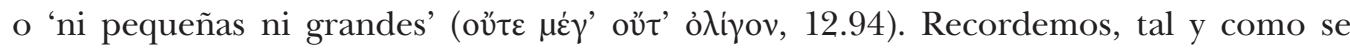
indicó antes, que este oleaje 'ruge' $(5.402,5.438,12.60)$ y produce 'estruendo' (Soṽ $\pi$ ov ä́ovбa, 12.202).

\section{La tormenta}

La combinación de los elementos anteriores, viento y oleaje, produce la 'tormenta', $\theta v \varepsilon \dot{\lambda} \lambda \alpha$, término que aparece en catorce ocasiones. En dos de ellas, los vientos se conjugan entre sí (5.315-318, 12.286-290), para, de ese modo, conferírsele en otros momentos

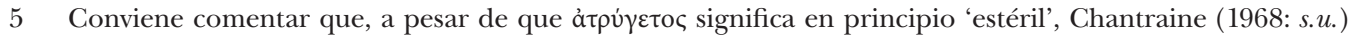

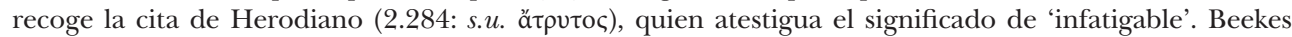
(2010: s.u.) también hace hincapié en esta posibilidad semántica, aunque la considera menos probable, en el sentido de 'que murmura con intensidad', en cuyo caso procedería de à- como intensivo y $\tau \rho \dot{\zeta} \zeta \omega$. 


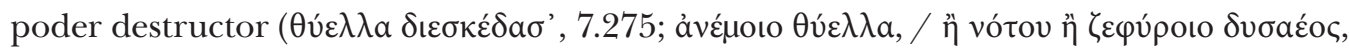

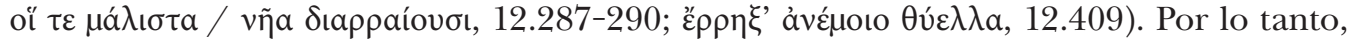

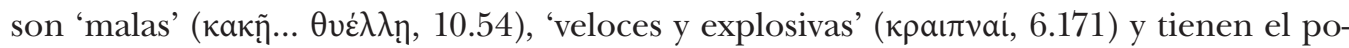

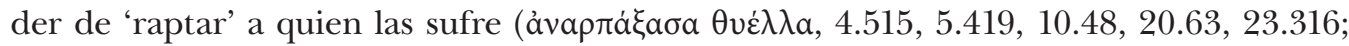

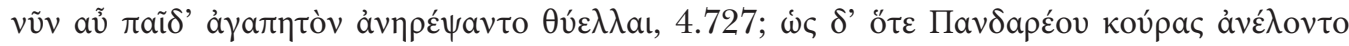

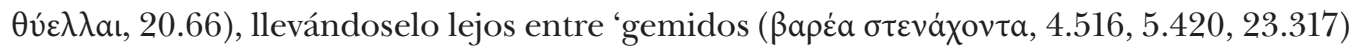

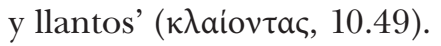

\section{La sonoridad de los salones palaciegos}

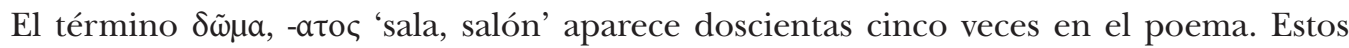
$\delta \dot{\omega} \mu \alpha \tau \alpha$ son habitualmente calificados como 'insignes' (ả $\gamma \alpha \kappa \lambda v \tau \dot{\alpha}, 3.388,3.428,7.3,7.46$ ),

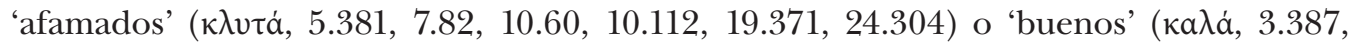
$8.41,10.13,10.252,15.454,16.109,17.264,20.122,20.319,22.495,24.361$ ). Dado que la Odisea es un poema que se ambienta en numerosas ocasiones dentro de recintos cerrados, la sonoridad de las salas de los edificios y también de la música que se ejecuta en su interior cobra gran importancia. Este tipo de espacios cubiertos suelen tener buena acústica, tal y como se ilustra con los momentos en que los aedos necesitan ser oídos por un público numeroso. Sin embargo, llama la atención que tan solo una vez en todo el poema se utiliza esta idea asociada a dicho sustantivo cuando son definidos como 'salo-

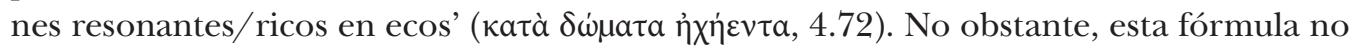
es exclusiva de este poema, puesto que se ha podido encontrar también en Ath. 5.14.26; hCer. 104; Hes. fr. 10a.46, 10a.86, 185.16; Eust. 1.146.26; Sch. in Iliadem (scholia vetera) 24.323b.2. Igualmente, creemos importante señalar que no aparece en ningún momento en la Ilíada, sin que haya motivos aparentes por los que sea así.

Otro término frecuentemente empleado en la Odisea para este tipo de edificios es

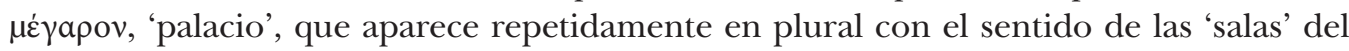
palacio y, por metonimia, el palacio en sí. Aparece doscientas once veces en el poema, de las cuales la mayoría se refiere a la localización espacial donde sucede algún hecho. Tan solo en ocho casos se refleja, de una manera indirecta, el sonido que en ellas se produce. Así, los pretendientes convierten la sala donde están alojados en un espacio

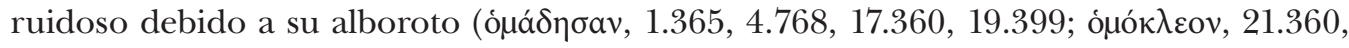
21.367, 22.211), frente a la única ocasión en que Femio llena con su música las salas del

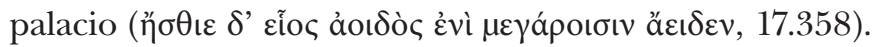

En relación con este último verso, podemos reseñar que, de los veinte ejemplos encontrados de ảoı́n', 'canto', este puede ser 'de inspiración divina' ( $\theta \dot{\varepsilon} \sigma \pi \imath v, 1.328,8.498)$, 'do-

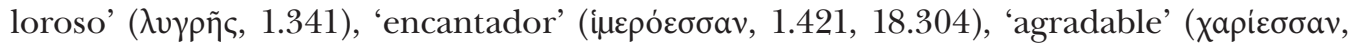

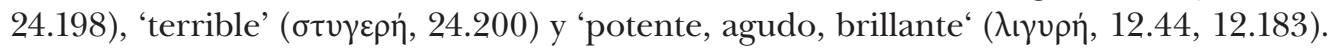

Sin diferencias sustanciales con ảot $\delta$, el texto presenta en tres ocasiones el término oй $(8.74,8.481,22.347)$.

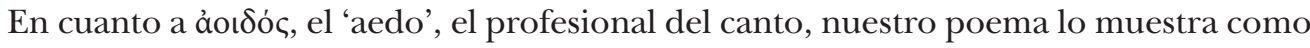

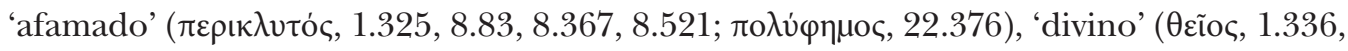




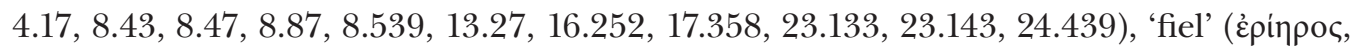
$1.346,8.62,8.471)$ y, de nuevo, 'de inspiración divina' ( $\theta \dot{\varepsilon} \sigma \pi \iota \varsigma, 17.385)$.

Los verbos ảot $\delta$ á́ (dos ejemplos) y ả $\varepsilon i \delta \omega$ (veintinueve ejemplos) son indicadores de una actividad musical en sí mismos. Sin embargo, esporádicamente son matizados

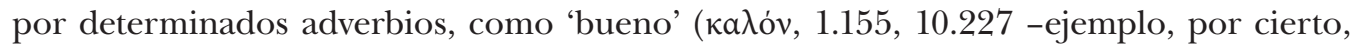

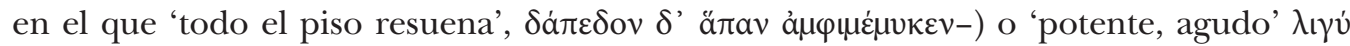
(10.254).

\section{La voz ${ }^{6}$}

Para el estudio de la voz, necesitamos desestimar los ochenta y tres ejemplos de usos de $\varphi \omega v \varepsilon \dot{\omega} \omega$, puesto que se emplea sin más contenido que el de indicar el inicio o el final del

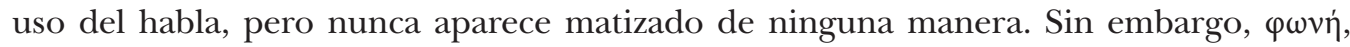
'voz' en sentido general, sí tiene más interés. De los diez ejemplos en que se emplea en el poema, se utiliza con Odiseo (19.381, 19.472), con Penélope (4.705), con mujeres (4.279) y con Atenea (24.530). Sin embargo, su uso crece ligeramente cuando se refiere a animales: a los cerdos de Circe (10.239), a las vacas (12.396), al águila, cuya voz es como la de los mortales ( $\varphi \omega v \tilde{n} \delta \dot{\varepsilon} \beta \rho o \tau \varepsilon \dot{n}, 19.545)$, y a la golondrina ('de voz muy dulce', $\pi \circ \lambda v \delta \varepsilon v \kappa \varepsilon \dot{\alpha}$ $\varphi \omega v \eta \dot{v}, 19.521)$.

Nos ha llamado mucho la atención que la fórmula $\lambda \varepsilon \pi \tau \alpha \lambda \varepsilon \dot{\eta} \varphi \omega v \eta$ 'voz fina/delicada', que aparece en $I l$. 18.571 referida al muchacho que interpreta la Canción de(l) Lino, no se encuentra, sin embargo, en la Odisea, sin que, en principio, pueda haber alguna razón sólida por lo que esto sea así.

Por otro lado, el grupo $\varphi \theta$ ó $\gamma \gamma \circ \varsigma / \varphi \theta$ / $\gamma \gamma \eta \dot{n} / \varphi \theta \dot{\varepsilon} \gamma \gamma$ oual, pertenecientes al campo semántico de la voz elevada y a veces ruidosa, se distribuye como sigue: los tres términos

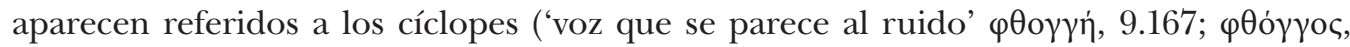
9.257; $\varphi \theta \dot{\varepsilon} \gamma \gamma$ oual, 9.497), mientras $\varphi \theta$ ó $\gamma$ oৎ se emplea una sola vez con las siervas de Penélope ('voz bulliciosa', 18.199). El verbo $\varphi \theta \varepsilon \dot{\gamma} \gamma$ oua aparece en una ocasión con Odiseo (21.192), pero es más frecuente con los marineros de Odiseo (10.228, 10.229, 10.255, 12.249), así como el sustantivo $\varphi \theta$ ó $\gamma o \varsigma$ con las Sirenas (12.41, 12.159, 12.198, 23.326). Podría decirse, por tanto, que este campo léxico, que, en principio, no debería suponer una sorpresa que se emplee con humanos en tanto en cuanto es una voz que genera algarabía, parece mucho más cercano a la descripción de la voz de los seres monstruosos o de humanos de un nivel inferior a los protagonistas.

El término ő $\psi$, 'voz bella y armoniosa', aparece empleado con mujeres de clase alta o con diosas (Casandra, 11.421; Penélope, 20.92; Musas, 24.60; Atenea, 24.535) y con Eu-

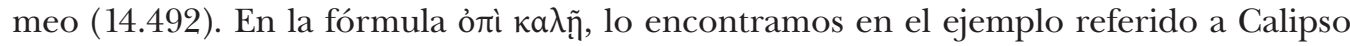

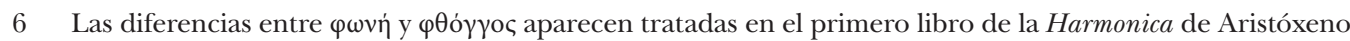
(s. IV EC) y, a partir de él, en algunos otros autores de la Antigüedad cuyos tratados musicales han llegado hasta nosotros. Ambos términos pueden referirse a la voz y al sonido indistintamente, aunque el segundo puede significar también 'nota'. Para un estudio pormenorizado de esta cuestión vid. Calero (2016: pp. 117-121), Garrido Domené (2016: pp. 159-267, 378-385). 
(5.61) y a Circe (10.221). Sin embargo, el grueso de ejemplos del uso de ö $\psi$ pertenece al episodio de las Sirenas (12.52, 12.160, 12.185, 12.187, 12.192), de donde uno podría deducir que, en cierto sentido, este término se aplica a un tipo de voz que puede resultar engañosa, un cebo para los incautos, especialmente cuando aparece en la fórmula

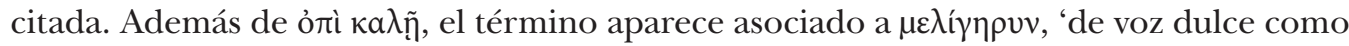
la miel' (12.187), y a кá $\lambda \lambda \mu$ ov, 'bello’ (12.192).

Por último, hay cinco ejemplos de $\mu$ o $\lambda \pi \eta$ 'canto que acompaña la danza' $(1.152,4.19$, 6.101, 21.430 -en que se pone énfasis en el juego y la danza-, 23.145) y dos de $\mu \varepsilon \dot{\lambda} \pi \omega$ 'cantar acompañando la danza' $(4.17,13.27)$.

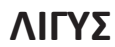

El campo semántico de $\lambda$ ı úc se relaciona con sonidos potentes, altos, claros y, en el caso de la voz y de los instrumentos, bien afinados. El tratado pseudo aristotélico de Aud.

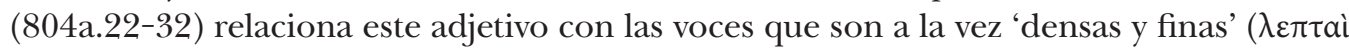

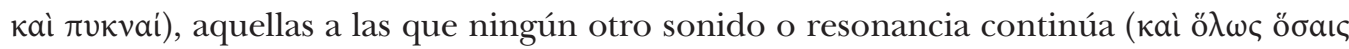

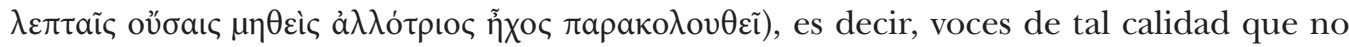
necesitan de instrumentos que las ayuden. En este sentido, la literatura griega antigua las vincula con voces que producen sonidos agudos, caracterizados por su 'fineza y pre-

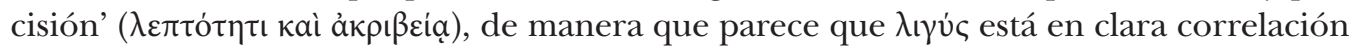
con el sonido claro, puro y sin cualidades que desagraden a quien escucha (Calero 2016: pp. 225-226).

Su etimología es desconocida (Chantraine 1968: s.u.; Beekes 2010: s.u.). Produce nombres compuestos que encontramos en poesía, como $\lambda \iota \gamma u ́ \varphi \theta o \gamma \gamma \circ \varsigma$, 'de voz potente, resonante, aguda', que en la Odisea aparece tan solo en 2.6, referido a los heraldos, o el también homérico $\lambda ı$ ıú $\omega v o \varsigma$, documentado solo en $I l .19 .350$ referido al halcón, aunque también aparece tanto en el repertorio épico (hMerc. 478, Hes. Th. 275, 518) como en el lírico (Sapph. fr. 30.8, Lyr.Adesp. fr. 7.1, Theoc. 12.7). Presenta relación léxica con los

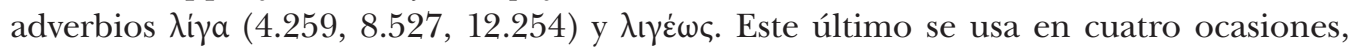
asociadas todas ellas al llanto ( $\kappa \lambda$ aĩov, $\kappa \lambda$ aĩ $)$, que es claro y potente (los tripulantes de la nave de Odiseo, 10.201; el alma de Agamenón, 11.391; Telémaco y Odiseo, 16.216; Penélope, 21.56).

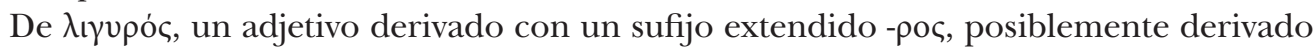
de -v- $\lambda$ ó (Chantraine 1968: s.u.; Beekes 2010: s.u.), contamos con un par de casos, vinculados ambos con las Sirenas y su canto, ảotón (12.44, 12.183).

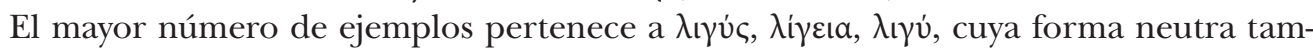
bién funciona como adverbio en algunos de ellos. Se emplea para determinar a un orador (20.274) o una Musa (2.62), pero contamos con cuatro casos en que se refiere al sonido del viento $(3.176,3.289,4.357,4.567)$ y, en mayor número y con toda lógica, cuando ilustra el sonido que emite la forminge $(8.67,8.105,8.254,8.261,8.537,22.332$, 23.133). 


\section{Conclusiones}

El análisis del mundo sonoro de la Odisea ha tenido que ceñirse a los campos léxicos estudiados en este artículo. Sin embargo, es necesario ampliarlo a otros más, que irán surgiendo en fases posteriores de la investigación.

Nuestro poema no distingue espacios físicos concretos, sino que las expresiones sonoras son intercambiables, funcionando en múltiples ocasiones como parte de las fórmulas. Es indudable que el uso metafórico de los atributos propios de los elementos naturales y del sonido que producen, incluso combinados entre sí, tiene un alto valor semántico en el poema. Sin embargo, la técnica formular parece desvestir de dicha carga semántica determinados ejemplos, supeditando el significado al uso de la fórmula. En todo caso, sí ayudan en numerosos puntos a ilustrar las emociones de nuestros personajes u objetos que los rodean, a modo de acotaciones teatrales, con el fin de dar más vivacidad y contextualizar más dramáticamente los distintos episodios.

Por último, sería interesante realizar un estudio similar con la Ilíada e incluso con el resto del repertorio épico, para ver hasta qué punto puede haber similitud entre todos ellos o si hay algún caso que caracterice a alguna de las obras concretas.

\section{Bibliography}

Allen, T. W. (Ed.). $\left(1985^{14}\right)$. Homeri opera. Odyssea (Vol. 1). Oxford: Clarendon Press.

Allen, T. W. (Ed.). (1985 $\left.{ }^{13}\right)$. Homeri opera. Odyssea (Vol. 2). Oxford: Clarendon Press.

Barker, A. (1989). Greek Musical Writings, I: The Musician and his Art. Cambridge: Cambridge University Press.

Beekes, R. (2010). Etymological Dictionary of Greek. Leiden - Boston: Brill.

Butler, S., \& Nooter, S. (Eds.). (2019). Sound and the Ancient Senses. London - New York: Routledge. Calero Rodríguez, L. (2016). La voz y el canto en la Antigua Grecia. Diss., Universidad Autónoma de Madrid [retrieved 17.09.2020 from https://repositorio.uam.es/handle/10486/676433].

Chantraine, P. (1968). Dictionnaire étymologique de la langue grecque. Histoire des mots (2 Vols.). Paris: Klincksieck.

Comotti, G. (1986). La música en la cultura griega y romana. Madrid: Turner Música.

Danek, G., \& Hagel, S. (1995). Homer-Singen. Wiener humanistische Blätter, 37, 5-20 [online available at https://www.oeaw.ac.at/kal/sh/whb37.htm].

Devine, K. (2014). Sound Studies. Grove Music Online. Oxford University Press [retrieved 17.09.2020 from https://doi.org/10.1093/gmo/9781561592630.article.A2258177].

Franklin, J. C. (2015). Kinyras. The Divine Lyre. Cambridge, MA - London: Harvard University Press; Center for Hellenic Studies [online available at http://nrs.harvard.edu/urn-3:hul.ebook:CHS_FranklinJ.Kinyras.2016].

García López, J. (1997). La mousiké tékhne en la Ilíada homérica. Molpé, aoidé kaì xopós. In F. Rodríguez Adrados (Ed.), IX Congreso Español de Estudios Clásicos (Vol. 2; pp. 103-107). Madrid: Ediciones Clásicas [reeditado en 1998 con el mismo título en L. Gil, M. Martínez Pastor, \& R. Ma Aguilar (Eds.), Homenaje al Profesor José S. Lasso de la Vega (pp. 363-368), Madrid]. 
García López, J., Pérez Cartagena, Fco. J., \& Redondo Reyes, P. (2012). La música en la antigua Grecia. Murcia: Universidad de Murcia.

Garrido Domené, F. (2016). Los teóricos menores de la música griega. Euclides el Geómetra, Nicómaco de Gerasa y Gaudencio el Filósofo. Barcelona: Cérix.

Hagel, S. (2008). Die Sänger aus musikarchäologischer Perspektive. In J. Latacz et al. (Eds.), Homer. Der Mythos von Troia in Dichtung und Kunst (pp. 106-111). München: Hirmer Verlag.

Henderson, I. (1957). Ancient Greek Music. In E. Wellesz (Ed.), Ancient and Oriental Music (pp. 336-403). London: Oxford University Press.

Hill, M. E. (2014). Soundscape. Grove Music Online. Oxford University Press [retrieved 17.09.2020 from https://doi.org/10.1093/gmo/9781561592630.article.A2258182].

Lord, A. B. (1960). The Singer of Tales. Cambridge, MA - London: Harvard University Press.

Michaelides, S. (1978). The Music of Ancient Greece. An Encyclopaedia. London: Faber and Faber.

Montero Honorato, Ma Pilar (1988). Homero y la música. Memorias de Historia Antigua, 9, 195-211.

Montiglio, S. (2019). The Sound of the Sirens between Sound and Sense. In S. Butler, \& S. Nooter (Eds.), Sound and the Ancient Senses (pp. 171-183). London - New York: Routledge.

Nooter, S. (2012). When Heroes Sing: Sophocles and the Shifting Soundscape of Tragedy. Cambridge: Cambridge University Press.

Nooter, S. (2017). The Mortal Voice in the Tragedies of Aeschylus. Cambridge: Cambridge University Press.

Pabón, J. M. (Transl.). (1982). Homero. Odisea (con introducción de M. Fernández Galiano). Madrid: Biblioteca Clásica Gredos.

Río Torres-Murciano, A. (2014). Música y épica de Homero a Estacio. Nova Tellus, 31(2), 185-198.

Sachs, C. (1940). The History of Musical Instruments. London: Dent \& Sons.

Sachs, C. (1943). The Rise of Music in the Ancient World. East and West. New York: Norton.

Saussure, F. de (1878). Mémoire sur le système primitif des voyelles dans les langues indo-européennes. Leipsick: Teubner.

Schafer, R. M. (1969). The New Soundscape. Scarborough, Ont. - New York: Berandol Music and Associated Music Publishers.

Schafer, R. M. (1970). The Book of Noise. Vancouver: Priv. print. by Price Print.

Schafer, R. M. (1977). The Soundscape: Our Sonic Environment and the Tuning of the World. Rochester, VT: Destiny Books.

Snoj, J. (2007). Music in Homer's Iliad. Musicological Annual, 43(1), 53-65.

Tapia Zúñiga, P. C. (2013). Vocabulario y formas verbales de la Odisea (Bibliotheca Scriptorum Graecorum et Romanorum Mexicana). México: UNAM.

Tapia Zúñiga, P. C. (Ed.). (2017²). Homero. Odisea (Bibliotheca Scriptorum Graecorum et Romanorum Mexicana). México: UNAM.

Valverde, M. (1997). El léxico musical en los Himnos Homéricos. In Actas del IX Congreso Español de Estudios Clásicos (Vol. II; pp. 259-264). Madrid: Ediciones Clásicas.

West, M. L. (1981). The Singing of Homer and the Modes of Early Greek Music. The Journal of Hellenic Studies, 101, 113-129.

West, M. L. (1994). Ancient Greek Music. Oxford: Clarendon Press. 
Prof. Dr. Luis Calero / luis.calero@uam.es

Department of Classical Philology, Lecturer of Greek

Autonomous University of Madrid, Faculty of Philosophy and Liberal Arts

Ciudad Universitaria de Cantoblanco, c/ Francisco Tomás y Valiente, 1, 28049 Madrid, Spain to relevant rights. 
